\title{
Wave Field Distributions for Toroidal Plasmas with nearly Circular Cross Sections
}
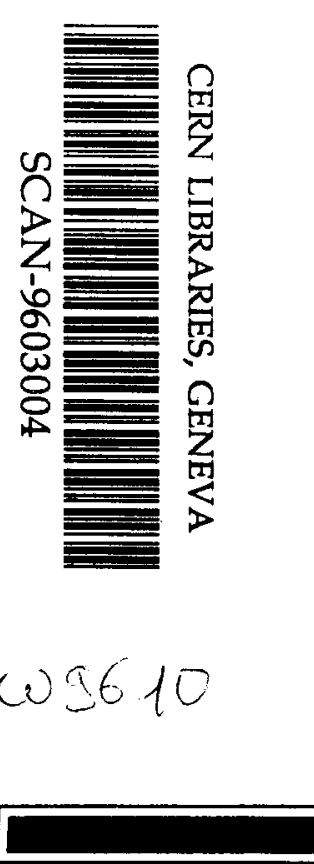
This document is intended for publication in the open literature. It is made available on the understanding that it may not be further circulated and extracts or references may not be published prior to publication of the original, without the consent of the Publications Officer, JET Joint Undertaking, Abingdon, Oxon, OX14 3EA, UK.

Enquiries about Copyright and reproduction should be addressed to the Publications Officer, JET Joint Undertaking, Abingdon, Oxon, OX14 3EA, UK. 
JET-P(95)61

\title{
Wave Field Distributions for Toroidal Plasmas with nearly Circular Cross Sections
}

\author{
T Hellsten ${ }^{1}$, J Källbäck ${ }^{1}$, L-G Eriksson. \\ JET Joint Undertaking, Abingdon, Oxfordshire, OX14 3EA, UK. \\ 1 Alfvén Laboratory, Royal Institute of Technocogy, S10044 Stockholm, Sweden, \\ (Euratom/NFR Fusion Association).
}

Preprint of a Paper to be submitted for publication in Physical Review Letters 


\begin{abstract}
Solutions of the wave equation in a geometry with stochastic ray trajectories have been considered. In particular we have studied the fast magnetosonic wave in the limit of weak damping. It is found that the often used assumption of a uniform wave energy density in the phase space for stochastic wave solutions does not hold, a conclusion which should be valid not only for applications related to wave propagation in plasmas. A new wave field model for stochastic fields has been obtained for tori with nearly circular cross sections.
\end{abstract}

Hamiltonian systems with stochastic ray trajectories appear in several disciplines of physics e. g. plasma physics, optics, acoustics, quantum mechanics. In connection with RF-heating and current drive in thermonuclear fusion plasma experiments, an interest has arisen in waves propagating in a toroidal cavity. The knowledge of the wave field strength and spectrum are required for calculating the power deposition and the effect of wave particle interaction. As the symmetry of the equilibrium is broken, e. g. going from a circular cross section to an elongated one, the ray trajectories can become stochastic. How the wave fields are distributed for such equilibria is an important question. Berry [1,2], when analyzing the wave function associated with stochastic motion, assumed for simplicity the Wigner function (local Fourier transform) of an eigenfunction to be isotropic in $\vec{k}$-space for any point in $\vec{x}$. On this basis McDonald developed a new method 
of calculating the wave field in the limit of weak damping for weakly inhomogeneous fields viz., the Wave Kinetic Equation, WKE $[3,4]$. This method is based on the fact that along the ray trajectories in the phase space the wave energy is constant. Assuming that the stochastic rays cover uniformly the propagating region in phase space a simple model of the stochastic wave field could then be obtained which did not require any detailed calculation of ray trajectories. Another approach to the problem was taken by Moreau et al [5], who expanded the wave fields in terms of eigenfunctions of a cylindrical equilibrium with a circular cross section, and derived a master equation for the wave spectrum. They concluded that, due to the large diffusion in the eigenmode expansion coefficients, the energy density is constant in phase space for lower hybrid waves propagating in tokamaks [6]. Later Kupfer et al applied McDonald's model to the fast magnetosonic wave for studying current drive in tokamaks [7]. In this letter we analyze the wave field in the limit of weak damping for a class of toroidal equilibria, which is of particular interest for the case of current drive by the fast magnetosonic wave. We have found that the eigenfunctions cannot be described as locally isotropic in $\vec{k}$. A new model to describe the "stochastic" wave fields for non-circular or toroidal equilibria in terms of "eigenfunctions" for a circular cylindrical equilibrium is given. Because we are not relying on any property specific for the fast magnetosonic wave we make the conjecture that in general the solution to a system with stochastic ray trajectories cannot be described by an assumption of homogeneous energy density in phase space.

In McDonald's model the wave field is represented by local Fourier series (transforms) varying in space, thus representing a function in phase space $(\bar{x}, \bar{k})$. When projecting a constant energy density from phase space into real space the energy density, $W(\vec{x})$, is obtained by summing (integrating) all propagating modes divided with their group velocity

$$
W(\vec{x}) \propto \sum_{m} \frac{U(\bar{x}, m)}{\mathrm{v}_{g r}},
$$


where $U(\vec{x}, m)$ is the wave energy density in phase space, $v_{\mathrm{gr}}$ the radial component of the group velocity, and $m$ the poloidal mode number. The group velocity is obtained from the dispersion relation of the fast magnetosonic wave

$$
k_{\perp}^{2}=(\omega / c)^{2} S-k_{\|}^{2}-\frac{(\omega / c)^{4}|D|^{2}}{(\omega / c)^{2} S-k_{\|}^{2}},
$$

where $S$ and $D$ are the diagonal and off-diagonal components of the perpendicular part of the dielectric tensor [8], $\perp$ and II denote the directions perpendicular and parallel to the equilibrium magnetic field, respectively. The propagating modes in a large aspect ratio torus with a nearly circular cross section are those for which $k_{r}^{2}>0$, where $k_{r}^{2} \approx k_{\perp}^{2}-m^{2} / r^{2}, r$ denotes the distance to the magnetic axis. According to the model described by Eq.(1) the wave field of the magnetosonic wave for large aspect ratio tori with constant plasma density should increase with minor radius because of the increasing number of propagating poloidal modes. This disagrees with numerical solutions for which still axially peaked wave field profiles are obtained, but not as peaked as those of a cylinder with a circular cross section $[9,10]$. The ergodic behaviour of the trajectories is manifested by the enrichment of the poloidal Fourier spectrum, but the peaked energy density profiles are not consistent with an isotropic energy density in the propagating region of the phase space. The energy density in phase space depends on how frequently the ray visits the volume element. Thus the assumption of an ergodic ray trajectory covering the region uniformly cannot hold for these equilibria.

Because of the discrepancy between the WKE model and the real solutions, we study the solutions of the fast magnetosonic wave in axially symmetric equilibria with the aim of finding a new model for "stochastic" wave fields. Since the toroidal angle is an ignorable co-ordinate we Fourier decompose it with respect to it and study the solution of a fixed toroidal mode number. The wave equation is solved with the global wave code LION [11], using the following parameters: $n=3 \times 10^{19} \mathrm{~m}^{-3}, T=5 \mathrm{keV}, f=33 \mathrm{MHz}$, $B_{0}=3.4 T, R_{0}=12 \mathrm{~m}$ and an aspect ratio $R_{0} / a=10$. We Fourier decompose the solutions in poloidal angle and average the square of the Fourier coefficients, $\left\langle\left|E_{\lambda, m}\right|^{2}\right\rangle_{m, s}$, over a radial wave length, over neighbouring $m$ and over equilibria with nearly similar plasma 
density. The radial dependency of some of the averaged Fourier coefficients are seen in Fig. 1 where we label the magnetic surface with $s=\sqrt{\psi / \psi_{a}}$, along a constant $\theta$-line $s$ is nearly proportional to $r, \psi$ denotes the poloidal magnetic flux and $\psi_{a}$ the value at the plasma boundary. The square of the Fourier coefficients decrease approximately as $1 / \mathrm{s}$ instead of being nearly constant in the region of propagation as the WKE model suggests i. e. where $r>m / k_{\perp}$. When the geometry deviates from a circular cylinder the equations in general become non-separable and the solutions become irregular. In Fig. 2 the poloidal mode spectrum is shown for different magnetic surfaces. The spectra near the magnetic axis are more narrow because of fewer propagating modes. Typically the wave spectrum excited by the antennae inside the plasma boundary for strong single pass damping has a half width $\Delta m=25$, which is more narrow than that of weak damping which in this case has $\Delta m=45$. The width of the wave spectrum for weak damping increases with plasma density contrary to that of strong single pass which is almost independent of the plasma density.

The radial dependence of the wave field can be understood by comparing the ray trajectories for a circular cylinder with those of a cylinder with slightly non-circular cross section or a torus. Since the corresponding ray trajectories for a single crossing are close to those of the circular cylinder, the radial variation of the poloidal Fourier modes should be close too. It is only after several crossings of the plasma the trajectories depart, when they depart the ray trajectories will then be represented by another poloidal mode number. Because of the similarities of the ray trajectories for a single pass we use a global expansion of the eigenfunctions in terms of the eigenfunctions of a circular cylinder instead of a local expansion to model the wave field. Further, we assume the radial wave length to be sufficiently short and the damping sufficiently large for there to be a resonating mode for a given frequency in the appropriate range and for every poloidal mode number for which the mode propagates. This leads us to the following model the stochastic wave fields

$$
E_{\lambda}=\sum_{m} \sqrt{C_{0} g(m) h(m, r)} \sigma_{1} \exp i\left(m \theta+\pi \sigma_{2}\right)
$$


where $\sigma_{1}$ and $\sigma_{2}$ are random numbers such that $\left\langle\sigma_{i}\right\rangle=0$ and $\left\langle\sigma_{i}^{2}\right\rangle=1$, the normalisation constant $C_{0}$ is determined by the total absorbed power, $\lambda$ is the direction orthogonal both to the magnetic field and the normal of the magnetic surface,

$$
\begin{gathered}
h(m, r)= \begin{cases}J_{m}^{2}\left(k_{\perp} r\right) & k_{\perp} r<C_{1}|m|+C_{2} \\
\frac{1}{\pi r \sqrt{k_{\perp}^{2}-m^{2} / r^{2}}} & k_{\perp} r \geq C_{1}|m|+C_{2} \\
\frac{2 A i^{2}(x)}{r\left|C_{3}\right|^{1 / 3}} & x>-1.2\end{cases} \\
x=\left|C_{3}\right|^{1 / 3}\left(r-r_{c}\right)-\frac{1}{4 r_{c}^{2}\left|C_{3}\right|^{2 / 3}, C_{3}=\frac{d}{d r}\left(k_{\perp}^{2}\right)_{r=r_{c}}+\frac{2 m^{2}}{r_{c}^{3}}-\frac{1}{2 r_{c}^{3}}, C_{1}=1.1143, C_{2}=0.9557,}
\end{gathered}
$$

$r_{\mathrm{c}}$ is the cut-off near the plasma boundary, $k_{\perp}=k_{\perp}(r, \theta)$ and $g(m)$ is a weight function. Unlike the true eigenmodes of a circular cylinder $h(m, r)$ has a $\theta$-dependence for finite aspect ratios through $k_{\perp}$. The summation is here taken over all waves propagating somewhere in the plasma. Outside the cut-off, $r=m / k_{\perp}$, the solution is approximated by WKB solution. Inside the cut-off and in a neighbourhood of it the solution is approximated by the square of the Bessel function, $J_{\mathrm{m}}$, at the inner evanescent region and with the square of the Airy function, $A i$, at the outer evanescent region. The approximation of the Fourier modes by Eq.(3) avoids the non-physical divergence of the WKB approximation at the cut-offs and is able to describe the wave field in the evanescent region. For comparison we have plotted $h(m, r)$ for $m=4$ in Fig. 1 using the same averaging as for calculating the averaged Fourier coefficients (averaging over neighbouring $s$ and $m$ ). In the region where the WKB approximation is valid good agreement between the model and the code is obtained.

For a cylinder with a circular cross section the Fourier modes are uncoupled and the weight function $g(m)$ is determined by the Fourier decomposition of the antenna current and coupling. For a non-separable system the Fourier modes couple and a much wider spectrum is observed, the averaged Fourier components decrease with the modulus of the wave number, but not monotonically. The modulus of the wave field square averaged by slightly different equilibria is given by 


$$
\left\langle\left|E_{\lambda}\right|^{2}\right\rangle=C_{0} \sum_{m} g(m) h(m, r)
$$

where the weight function $g(m)$ be found by comparison between $\langle F(s)\rangle=\int_{V(s)}\left\langle\left|E_{\lambda}\right|^{2}\right\rangle d V$ calculated with the LION-code and $\langle F(s)\rangle$ calculated with Eq. (4). For large aspect ratio, elliptic Solovev equilibria with a constant plasma density $g(m) \approx|m|^{-x}$ for $m \neq 0$ with $\kappa=0.65$ and $g(0)=1$ approximate $\langle F(s)\rangle$ well as can be seen in Fig. 3. For comparison $\langle F(s)\rangle$ is also shown assuming the wave energy to be constant in the region of the phase space where the waves propagate.

The stochastic wave field model given by Eq.(4) should approximate the wave field averaged over similar equilibria. For low density, corresponding to long wavelength, only a few poloidal Fourier modes appear, a stochastic wave field description becomes then less meaningful. As the density increases the wave length decreases and the deviations of the flux surface averaged wave field from individual solutions become less and the model becomes more appropriate. For the comparisons of $\langle F(s)\rangle$ shown in Figs. (3-5) we have averaged the square of the wave fields over equilibria with slightly different density.

The number of poloidal modes increases with density, variation of the density profile becomes therefore a critical test for the applicability of the model. A density profile decreasing with radius and one increasing with radius are used for this test. As can be seen in Fig. 4 the model describes the change in the wave fields due to the change in the density profile rather well. For tori with small aspect ratios, $k_{\perp}$ will vary significantly along the outer flux surfaces. Comparison of $\langle F(s)\rangle$ for a small aspect ratio equilibrium with constant density is shown in Fig. 5.

To test the poloidal distribution of the wave field we compare $\left\langle F_{m}(s)\right\rangle=\int_{V(s)}\left\langle\left|E_{\lambda}\right|^{2}\right\rangle \cos m \theta d V$ calculated with the LION-code and with the model for up down symmetric equilibria. As the deviation from a circular cross section becomes large 
and the aspect ratio small the deviation of $\left\langle F_{m}(s)\right\rangle$ between Eq. (4) and the LION-code increases.

A stochastic model of the wave field for short wave length modes in the limit of weak damping has been developed. It is applicable to wave propagation in toroidal geometries for a rather wide range of equilibria. From the comparison shown in Figs. 4 and 5 we conclude that the model described by Eq.(4) with the same weight function $g(m)$ is able to approximate "stochasticity" caused by ellipticity as well as by toroidicity. The model describes the modulus of the wave field averaged over nearly similar equilibria e. g. for which the plasma densities varies slightly. In addition to treatment near the cut off and in the evanescent regions our formula deviates from the WKE used by Kupfer $e t$ al [7] by the $1 / r$ term in the $h(m, r)$ function and that the weight function $g(m)$ is not constant. Both of the latter effects give rise to a more centrally peaked wave field, but not as peaked as for a circular cylinder. The general conclusion one can draw from our findings are that the often used assumption of a constant wave energy density in the phase space does not hold. Due to the general nature of the problem the analysis should have implications on stochastic solutions of the Helmholtz equation as well as to propagation of other kinds of weakly damped plasma waves.

ACKNOWLEDGEMENT: This work has been supported by the European Communities under an association contract between Sweden and EURATOM and under JET study contract No. JT4/13744. The authors acknowledge CRPP, Lausanne, for having made the LION code available and the Swedish National Supercomputer Centre for the access of the CRAY-YMP.

\section{REFERENCES}

[1] Berry, M.V., J. Phys. A, 10(1977)2083.

[2] Berry, M.V., Philos. Trans. Roy. Soc. London. Ser. 287(1977)237. 
[3] McDonald, S, W. and Kaufman, A. N., Phys. Rev. Lett. 42(1979)1189.

[4] McDonald, S. W., Phys. Rep 158(1988)337.

[5] Moreau, D. et al, Plasma Physics and Controlled Fusion 31(1989)1895.

[6] Kupfer, K. et al, Physics of Fluids B5(1993)4391.

[7] Kupfer, K. et al, Phys. of Plasmas 1(1994)3915.

[8] Stix, T. H., Waves in Plasmas, American Institute of Physics, New York, 1992.

[9] Hellsten, T. and Villard, L., Nucl. Fusion 28(1988)285.

[10] Hellsten, T. and Eriksson, L.-G., Nucl. Fusion 29(1989)2165.

[11] Villard, L., et al, J. Computer. Phys. Reports 4(1986)95. 


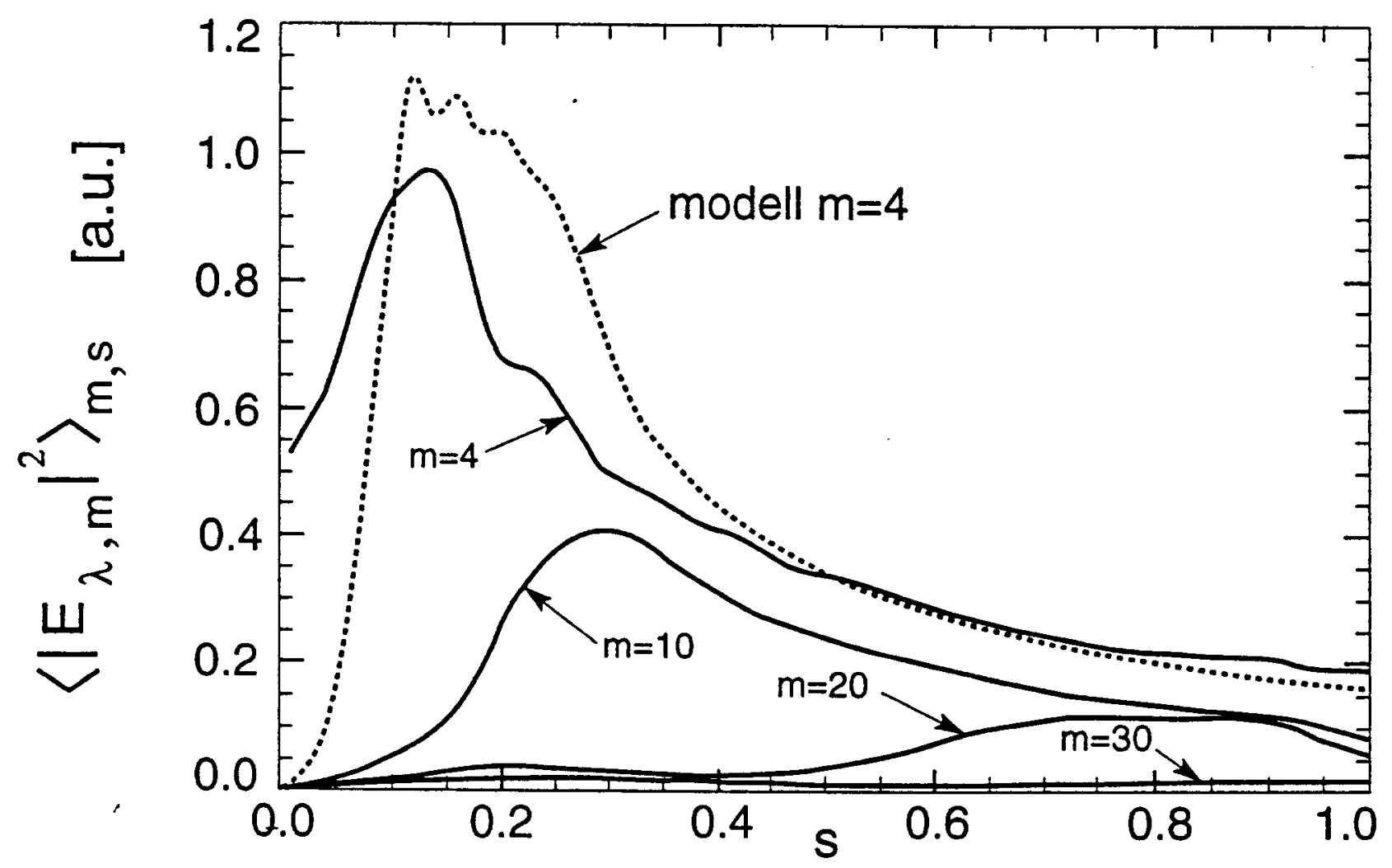

Figure 1. The radial dependence of the poloidal Fourier coefficients of the wave field calculated for a Solovev equilibrium with ellipticity $=1.26$. The square of the coefficients are averaged for neighbouring $m$ and $s$. Dotted curve is Eq.(3) normalised at $s=0.5$. 


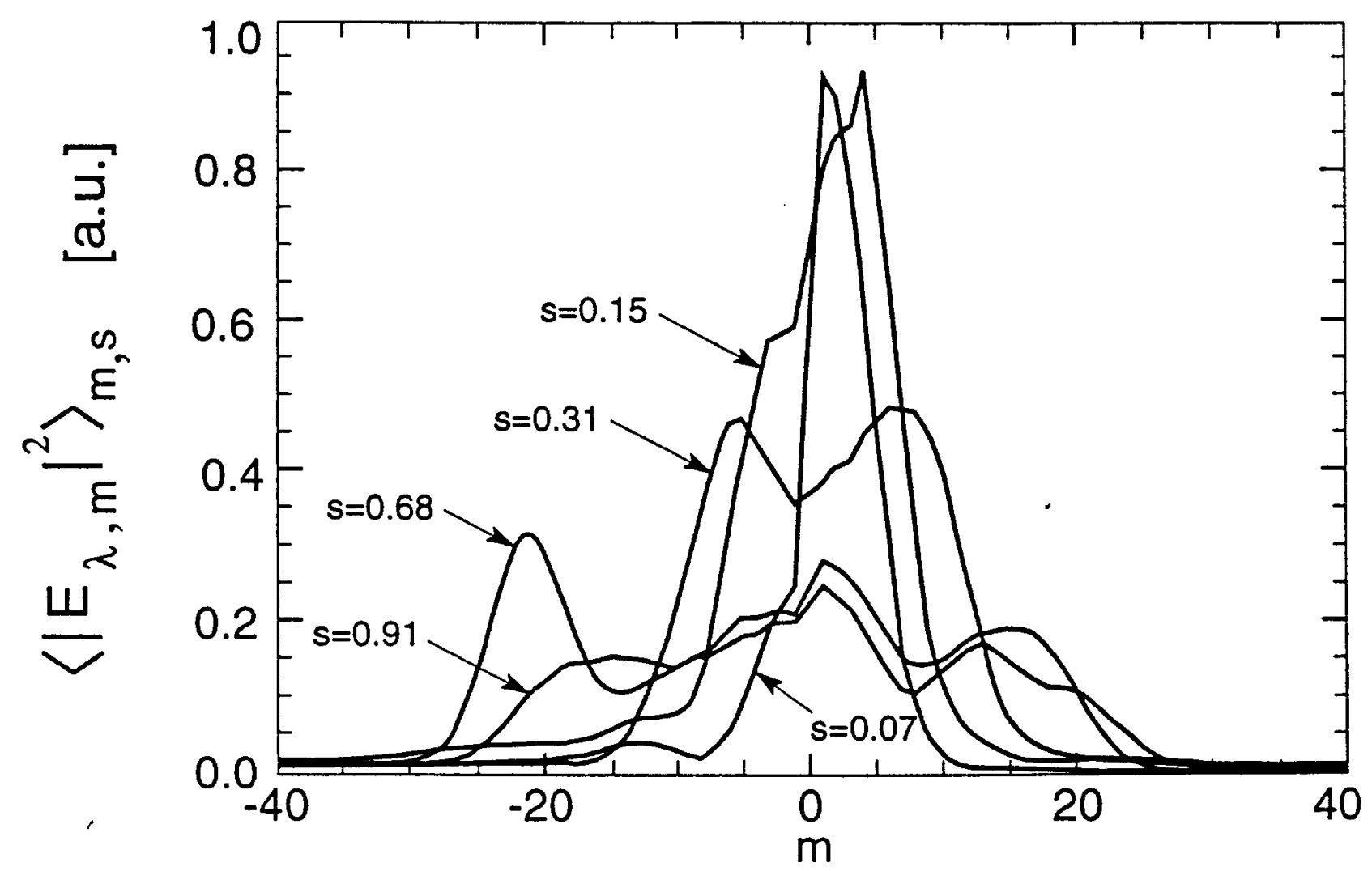

Figure 2. Poloidal mode number spectrum. The numbers indicate the $s$ value of the surfaces. 


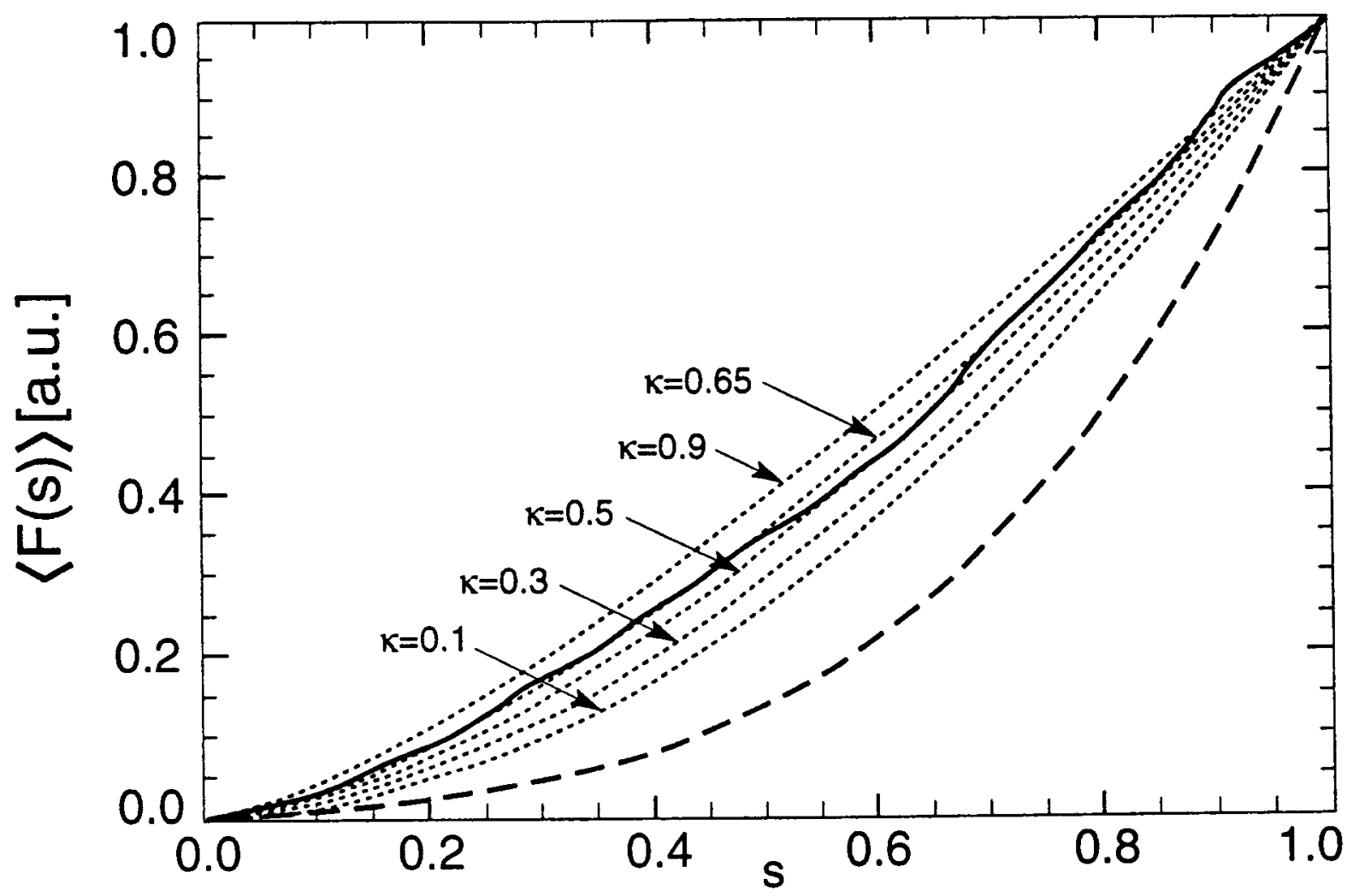

Figure 3. The flux surface integrated wave field $\langle F(s)\rangle$ for an elliptical Solovev equilibrium with constant density profile, ellipticity $=1.26$, and aspect ratio of 10. Dotted lines Eq.(3) with different $\kappa$, full line the LION code and the dashed line for constant energy density in the propagating region of the phase space. 


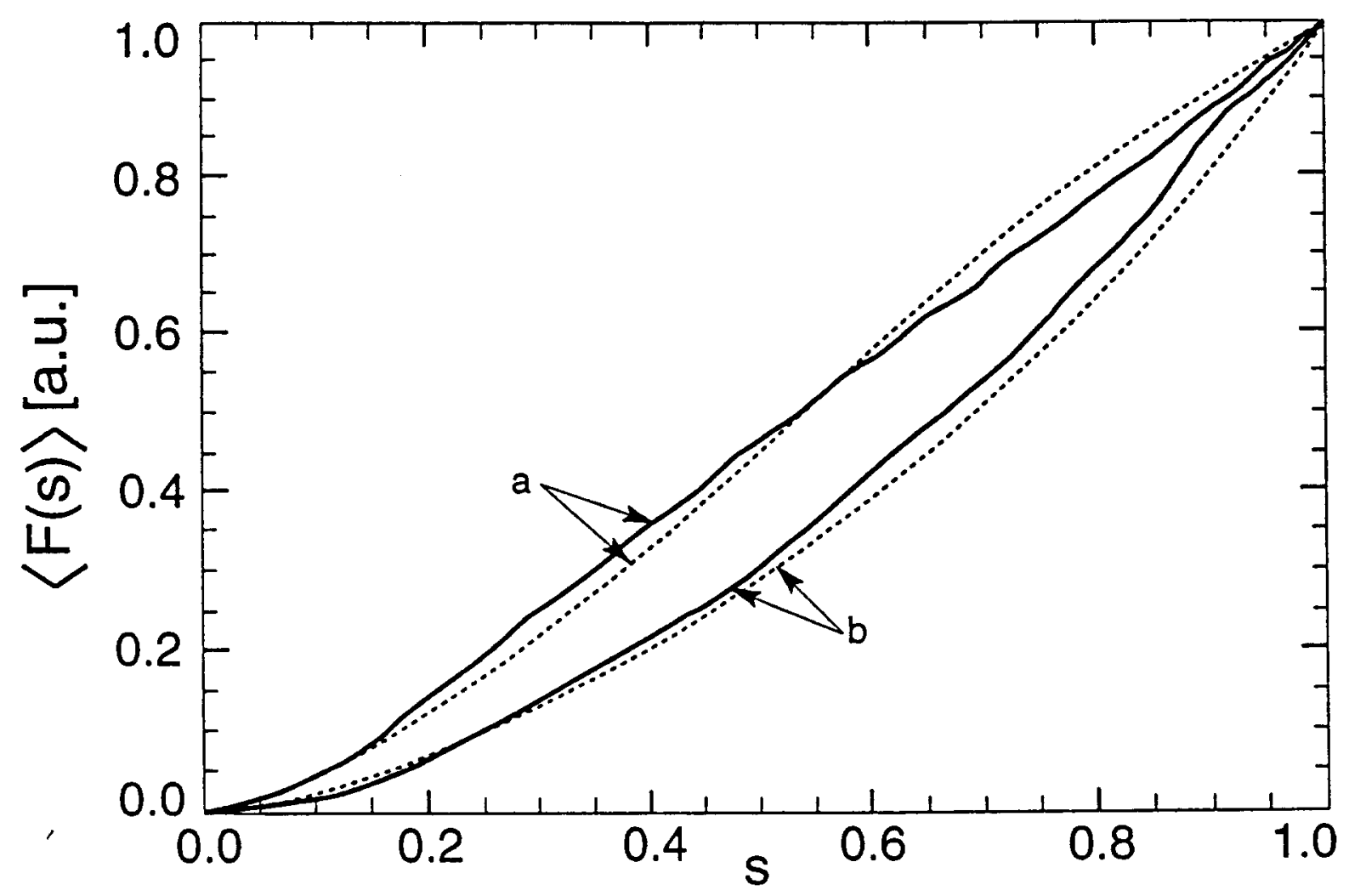

Figure 4. Comparison of the flux surface integrated wave field $\langle F(s)\rangle$ for elliptic equilibria with large aspect ratio, full lines LION code and dotted lines Eq.(3), (a) with a density profile $n=n_{0}\left(1-0.9 s^{2}\right)$ and (b) with a density profile $n=n_{0}\left(1+4 s^{2}\right)$. 


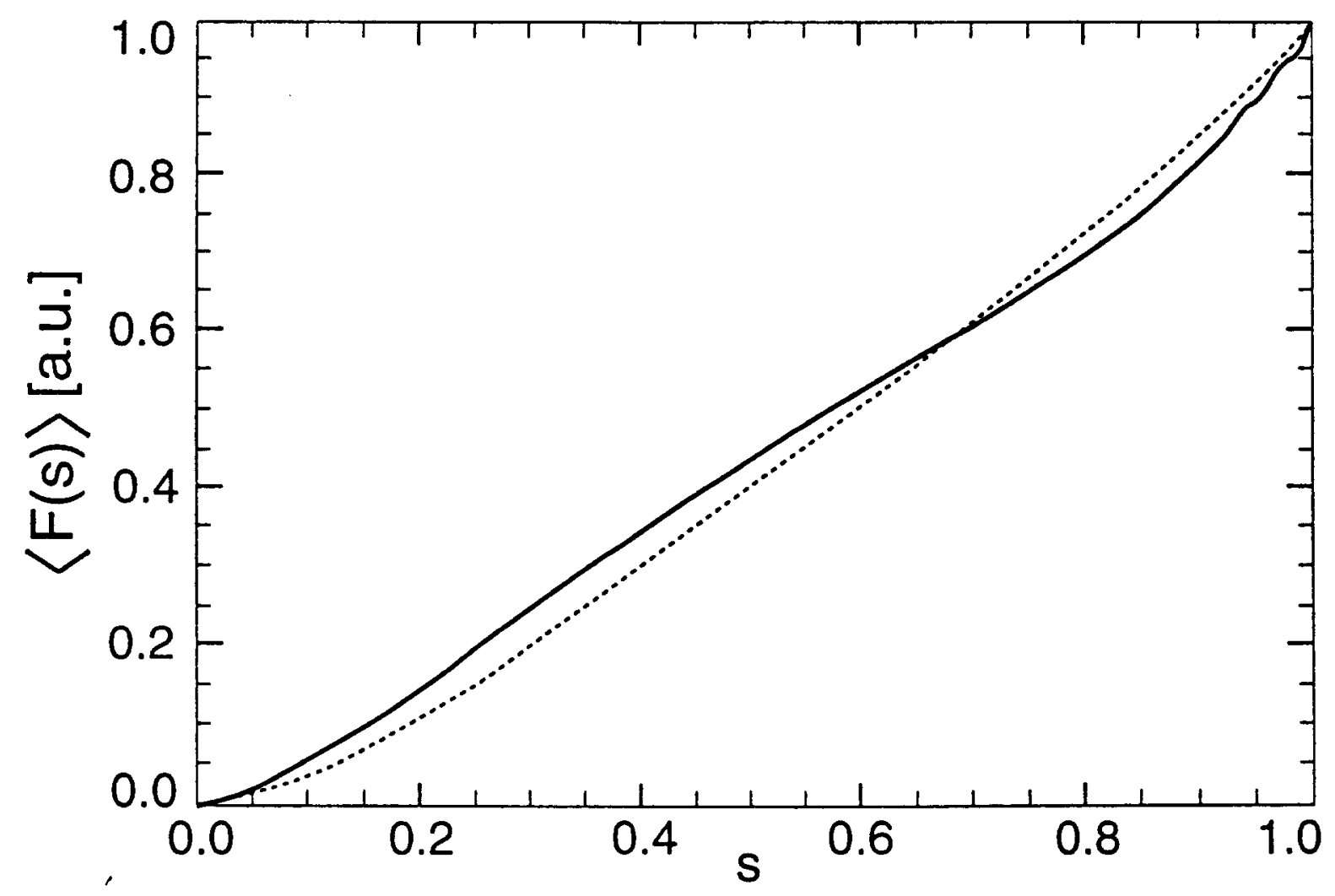

Figure 5. Comparison of the flux surface integrated wave field $\langle F(s)\rangle$ for an equilibrium with a circular cross section and an aspect ratio of 3 . Full line LION code and dotted line Eq.(3). 
